\title{
Cavitation treatment of water from a wastewater treatment plant
}

\author{
Donka Stoeva ${ }^{1, *}$, Apostol Simitchiev ${ }^{2}$, and Hristo Hristov ${ }^{1}$ \\ ${ }^{1}$ University of Food Technologies, Plovdiv, Bulgaria, Department of MAFFI, Maritza 26, blvd.
}

\begin{abstract}
Hydrodynamic cavitation damages the apparatus by intensive cavitational erosion of the elements in a hydro system. Being acquainted with the mechanisms of effect, hydrodynamic cavitation can be used to intensify technological processes in different industrial areas. The aim of the present work is to investigate the possibilities using hydrodynamic cavitation for wastewater treatment from an urban treatment plant. To intensify the purifying process series of trials, at different levels of intensity of the electrical field in the zone of cavitation, were performed. Additionally presented, the electrical field assists in forcing the process of silver ions separation with the purpose of microbiological purification of the flowing water. Here we present the results of experimental studies conducted at various times and electrical fields intensity. These trials substantiate the idea of the microbiological purification of wastewaters via hydrodynamic cavitation and represent a huge range of opportunities for applying this method in various industrial fields. The basic criterion that supports our statement is the enormous drop of the microbe number by increasing the cavitation treatment time.
\end{abstract}

\section{Introduction}

In the process of use in industry, the water is usually enriched with impurities of different nature and dispersion, as a result of which the used water has to be removed from the production process and released into the environment, i.e. wastewater is produced. The resulting need for the treatment of large quantities of waste water before their release into the environment also conditioned the rapid development of wastewater treatment technology, together with water preparation, making them necessary for modern production and life. The new European directives on environmental protection require the introduction of new systems for protection of the country's water resources. This is necessary because of the need to preserve aquatic ecosystems fit for use in diverse human activities. Radial precipitators which provide a relatively good treatment effect are used for medium and large treatment plants. Radial precipitators are reinforced concrete with a circular cross section. Their diameter is usually in the range of 12 to $60 \mathrm{~m}$, depth of the working part from 1.5 to $5 \mathrm{~m}$, and the ratio of the diameter to the working height is $\mathrm{D}=(6 \div 30) \mathrm{H}$. As with the other precipitators, the main elements of the radial ones are an inlet distribution device, a working part, a ring trough for the settled water and a sludge chamber. In practice,

* Corresponding author: bodurova@gmail.com 
according to the way of water supply, different types of radial precipitators are used. The most frequently used in practice are: standard radial precipitators, radial precipitators with peripheral water supply, thin-layer precipitators. When choosing a suitable type of precipitator, it is advisable to take into account a set of factors, including the performance of the wastewater treatment plant, technological scheme of treatment, geological conditions, groundwater level, etc. In the current norms for design of sewerage systems in Bulgaria, the sizing of all types of primary precipitators is recommended according to the generalized method, based on experimental studies of the kinetics of precipitation of solutes. The regulatory framework also states that precipitators for reagent water purification should be designed with built-in reaction chambers. According to the document, the type of precipitator is determined on the basis of a feasibility study. The precipitator is dimensioned with experimentally determined values of the sedimentation rate as predetermined water stay and depth of the sedimentation part. The turbidity of the precipitated water is required not to exceed values higher than $8 \div 12 \mathrm{mg} / \mathrm{dm}^{3}[1,2,3]$.

Biologically purified waters contain difficult degradable organic substances, such as pesticides, detergents, phenols, mineral oils, etc. They contain biogenic elements such as nitrogen and phosphorus and bacterial contamination. It is proven that developed hydrodynamic cavitation can be used to intensify technological processes in water preparation, the production of high alcoholic and non-alcoholic beverages, receiving stable emulsions, etc. $[4,5,6]$.

Wastewater in auxiliary productions consist an unstable composition of microorganisms. Microorganisms are constantly under the influence of various environmental factors which affect their growth and development. Microorganisms stand on high pressure and mechanical shocks. However, there is a pronounced selectivity of the bactericidal action of ultrasound, which is due to the morphological features and physiological state of microorganisms. The most important role plays the cell shell, and then - the shape and size of the cells. The highest sensitivity to ultrasound possess filamentous bacteria, smaller - rod-shaped bacteria, and the most resistant are the cocoforms (coccal bacteria) [7].

Cavitation bubbles are filled with liquid fumes, and when they suddenly shrink into the liquid, a huge pressure occured around them. When the microorganisms, contained in the water, fall into a cavitation zone, they lose their viability. Moreover, hydrogen peroxide and nitric dioxide appears in the fluid, which interacts with the water and forms nitric and nitrous acids. Most often, it results in a torn cell shell of microorganisms. With the phenomenon "Cavitation", the "hit" on the bacterium cell comes from the outside. $[8,9]$.

\section{Materials and methods}

\subsection{Laboratory stand for cavitation treatment of wastewater}

The laboratory stand shown on Fig. 1 was used for the study $[1,2,3]$. The cavitator also provides a constructive possibility to place a silver plate in the area of developed cavitation, as well as to feed constant voltage in order to more intensively release silver ions. The cavitator design allows the possibility to adjust the intensity of the hydrodynamic cavitation process. This can be achieved by changing the pressure and flow in the system, as well as by changing the geometric parameters of the flow part of the cavitator. The minimum crosssection is accepted for defining geometric parameter. A bacterium wall outlines the borders of the microbe body. It contacts the environment and has a thickness of $5 \div 30 \mathrm{~nm}$. The bacteria wall is two-layered. Its internal layer consists of spherical macromolecules at a diameter of $12 \div 14 \mathrm{~nm}$. It is determined that between those macromolecules there are pores 
which are up to $1 \mathrm{~nm}$ in size. They could naturally hold non-dissolved gas that can be a basis of a cavitation core formation. [4]. Supersonic waves have a strong influence, which at certain conditions, may lead to immediate cell destruction. Often the cell membrane is the most vulnerable part. Supersonic as a sequence of cavitation acts destructively toward all groups of microorganisms-mushrooms, bacteria, actinomicets and viruses. [8, 9, 10]

The aim of the present work is to investigate the possibilities using hydrodynamic cavitation for wastewater treatment from an urban treatment plant. To intensify the process of purification, electrical field is created and the field has different intensity upon silver plates placed in the cavitation zone. The experimental studies are conducted on a stand where hydrodynamic cavitation is performed in the zone of a cavitator with especially projected protracted part. The diagram of the stand is on fig.1a and 1b. Before each trial starts, we take a $10 \mathrm{ml}$ control water probe from the reservoir (non-cavitated water). It is the base to compare the probes of the cavitated water during the different time intervals. Vacuum followed by overpressure reacts as effectively as high-pressure the stream is. Microorganism destruction by cavitation with damage of their cell membrane is based on application of that high-impact effect. $[11,12]$

\subsection{Preparing the materials for an experiment}

A closed-type experimental installation scheme was used to determine the effectiveness of cavitational effects on wastewater. It consists of a cavitator, centrifugal pump, tank, pipeline, measuring instruments and adjusting taps (Fig. 1). The microbiological analysis of the water samples is carried out in an accredited microbiological laboratory of an Urban Treatment Plant. The cavitator shape is designed in such a way as to enable to create maximum circulation zone and also to obtain intensive cavitation. [11]

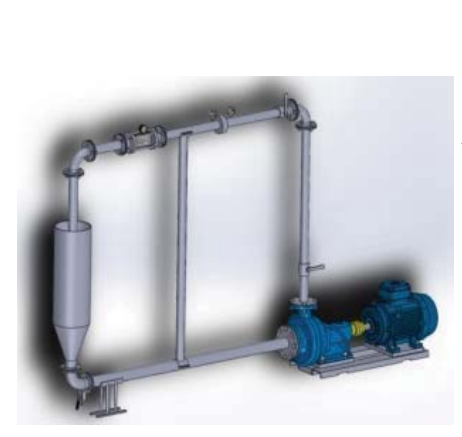

a) 3D Model

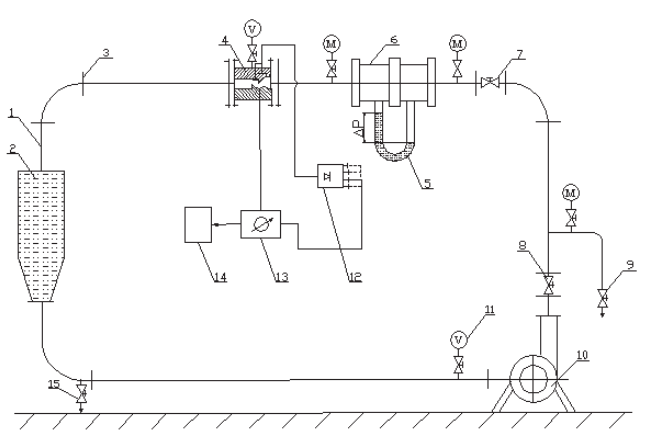

b) Scheme

Fig. 1. Scheme of laboratory installation

1 - tubing system; 2 - reservoir; 3 - flange; 4 - cavitator; 5 - pressure gauge; 6 - diaphragm; 7 control valve; 8 - injection valve; 9 - release valve; 10 - centrifugal pump; 11 - vacuum gauge; 12 rectifier; 13 - digital multimeter; 14 - computer; 15 - valve to take tests

A silver plate, which released silver ions during the cavitation process, was inserted into the cavitator in order to intensify the waste water purification process. The location of the silver plate was chosen so that it was in the impact zone on the wall of the detached jet, where the strongest cavitation erosion was expected. This ensures the release of silver ions into the liquid. In order to intensify the process of ion release $\left(\mathrm{Ag}^{+}\right)$, a constant voltage of different value was applied to the silver plate (Fig. 1). The voltage supplied on the silver plate in the cavitator was in the range from $17 \mathrm{~V}$ to $27 \mathrm{~V}$. $[12,13,14]$. The experimental 
studies were conducted on a stand where hydrodynamic cavitation is performed in the zone of a cavitator with especially projected protracted part.

Before each trial starts, we take a $10 \mathrm{ml}$ control water probe from the reservoir (noncavitated water). It is the base to compare the probes of the cavitated water during the different time intervals. The place of hitting the cavern onto the wall of the streamed object (vessel) or against the microbe cell is charged with high pressure. Upon the surface of the cell wall we provoke hydraulic impact by alternation of vacuum and high pressure that attends the closing of a cavitation cavern. The high speed of the stream performed at the cavern destruction is the reason for the cavitation mechanic act and for the microorganism cell wall destruction. The exact reason for microorganism destruction is the damage of their cell wall. Another product of the hydrodynamic cavitation in the zone of cavitational erosion is the separation of silver ions that have strong bacterium effect and force the microbiological water purification.

\subsection{Measurement method}

In all variants of the experiments, the pressure and flow rate in the system shall be measured. The cavitation time shall also be measured and a microbiological analysis of the water samples shall be carried out, determining the microbial number for each attempt. The processing time at which the best effect is achieved is also determined in an experimental way. The microbial number is the basic parameter that characterizes the degree of water purification. Criterion for the effectiveness of cavitational influence on wastewater is the decrease of the microbiological parameter - microbial number. The microbial number is defined as the total number of mesophilic heterotrophic microorganisms [cfu/ml- colony forming unit per ml.]. These are the most common microorganisms in water. Mesophilic microorganisms are those that live in average temperature limits. The microbial number is a parameter of optimization (dependent variable)- this is the resulting reaction from the complex impact of the input factors on which depends the behavior of the whole system. The variation of the microbial number indicates the influence of factors on the cavitational wastewater purification process. Microbiological water testing begins immediately after taking the samples and was carried out in accordance with the requirements of the Hygiene and Epidemiology Inspection [15].

\subsection{Cavitational effects mechanism on micro-organisms}

When microorganisms contained in water fall into the developed hydrodynamic cavitation zone, they are subjected to its destructive action. The surface of microorganisms is destroyed due to the impact of cavitation caverns on the cell wall and their erosion action. The number of killed microorganisms is greatly increased with the time of cavitation impact. Pressure and speeds pulses are the result of changing geometric conditions in the cavitation zone. These influences are decisive in the modeling of the shape of the flowing part. The killing of microorganisms, which leads to the purification of wastewater and other liquids from microbiological contaminants, is a result of the cavitation impact. $[4,6,14$, $16]$.

Studies have been carried out on the magnitude of cavitational destruction in superplastic materials to which we can also refer the cell walls of microorganisms. There is a link between the force of the closing cavernous and the destruction of erosion. At the diameter of the destruction $60 \mu \mathrm{m}$ the corresponding depth is $650-700 \mathrm{~nm}$ and the depth varies depending on the density of the material. Therefore, the strength and magnitude of the cavitational impact is sufficient to tear the microorganism's cell wall. 


\section{Results and analysis}

\subsection{Carried out trials results}

The conditions under which the experiments were performed were identical for all the studies. Initial temperature of the the treated water $20^{\circ} \mathrm{C}$ and air humidity of $55 \%$. The laboratory stand was disinfected before each experiment to ensure the accuracy of the result.

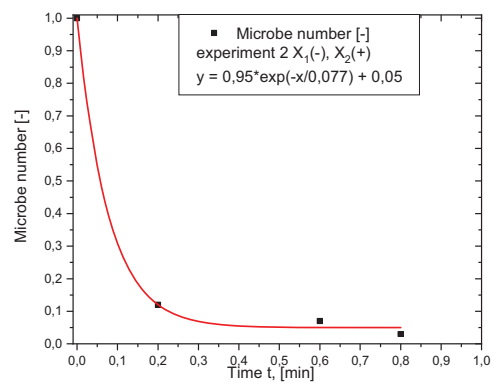

Fig. 2 Graph for the reduction of the microbial number during cavitation processing in an dimensionless form

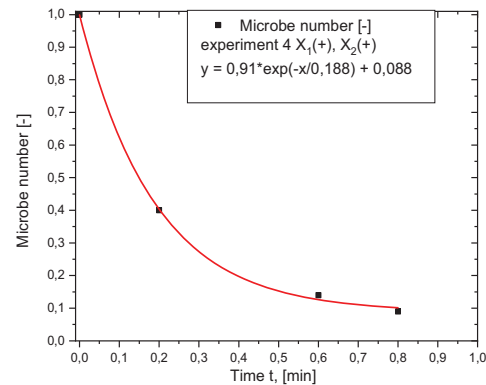

Fig. 3 Graph for the reduction of the microbial number during cavitation processing in an dimensionless form

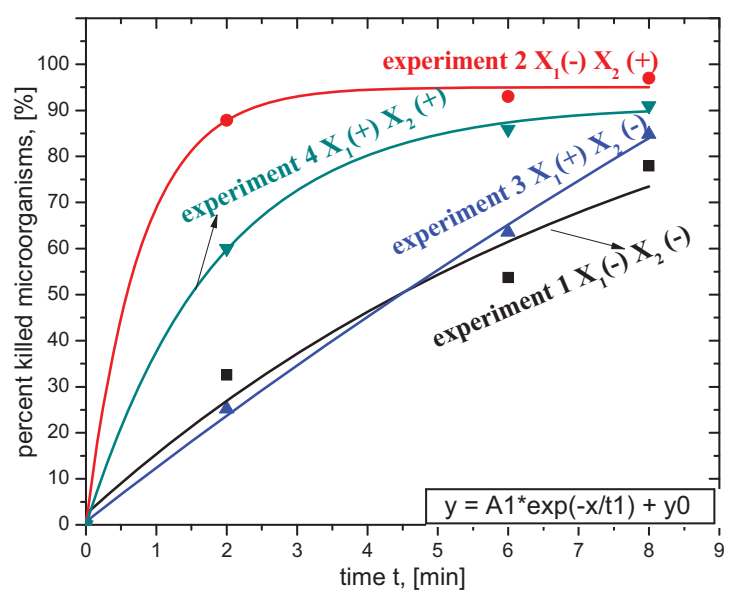

Fig.4 Increasing of the percentage of microorganisms - killed during treatment

Figure 2 presents the decrease in microbial number during cavitation treatment when the time factor $\left(\mathrm{X}_{1}\right)$ is at lower level, and the pressure $\left(\mathrm{X}_{2}\right)$ is at higher level. Figure 3 presents the decrease of the microbial number during cavitation treatment when factors time $\left(\mathrm{X}_{1}\right)$ and pressure $\left(\mathrm{X}_{2}\right)$ are at their higher level. Table 1 presents the coefficients values in the equation, obtained when the curves for the Modification of the percentage of killed microorganisms in Fig. 4 were drawn. The photos of the cavitator are shown on fig.5,6,7.

Table 1 Values of the coefficients in the equation

\begin{tabular}{|l|l|l|l|}
\hline Equation & Y=A1*exp(-x/t1)+Y0 & & \\
\hline Adj. R-Square & 0.8927 & 0.99645 & 0.997 \\
\hline & & Value & Standard error \\
\hline
\end{tabular}




\begin{tabular}{|l|l|l|l|}
\hline \multirow{5}{*}{ Experiment 1 } & Y0 & 117.944 & 126.05 \\
\cline { 2 - 4 } & $\mathrm{A} 1$ & -115.56 & 121.92 \\
\cline { 2 - 4 } & $\mathrm{t} 1$ & 8.377 & 14.5 \\
\hline \multirow{3}{*}{ Experiment 2 } & $\mathrm{Y} 0$ & 95 & 1.97 \\
\cline { 2 - 4 } & $\mathrm{A} 1$ & -95.01 & 3.39 \\
\cline { 2 - 4 } & $\mathrm{t} 1$ & 0.775 & 0.139 \\
\hline \multirow{3}{*}{ Experiment 3 } & $\mathrm{Y} 0$ & 346.98 & 348.6 \\
\cline { 2 - 4 } & $\mathrm{A} 1$ & -346.3 & 347.4 \\
\cline { 2 - 4 } & $\mathrm{t} 1$ & 29.09 & 33.57 \\
\hline Experiment 4 & $\mathrm{Y} 0$ & 91.09 & 1.78 \\
\cline { 2 - 4 } & $\mathrm{A} 1$ & -91.025 & 2.5 \\
\cline { 2 - 4 } & $\mathrm{t} 1$ & 1.88 & 0.147 \\
\hline
\end{tabular}

\subsection{Algorithm for conducting a full factor experiment}

In the carried out single-factor experiments and on the basis of the publications existing so far, concerning the problem, were identified the following important independent factors on the degree of cavitation disinfection: processing time and applied voltage to the silver plate. It was conducted a complete factor experiment - type $2^{2}$ with three repetitions at each point of the plan (from the factor space) in order to obtain an adequate regression equation.

\subsubsection{Determination of significant influencing input factors}

The factors influencing the process are independent measurable quantities that can occupy specific values under certain conditions. They were selected on the basis of expert evaluation, initial one factor experiments and a detailed literature study on the topic In cavitation erosion, silver ions are released from the plate placed in the cavitator. In order to intensify the process of separation of silver ions, a constant voltage is applied to the plate. The natural values of the studied factors are presented in table 2 . The full factor experiment identifies the significant influencing input factors on the accepted optimization parameters $[2,5,14] . \mathrm{X}_{1}$-t $[\mathrm{min}]$-processing time, $\mathrm{X}_{2}-\mathrm{U}[\mathrm{V}]$-voltage, are variable dimensions, which show the environment influence of the study object. $[3,8,17]$. Independent factors that influence the cavitation process of water purification are:

1. Cavitation time $X_{1}$.

2. A voltage shall be fed into the area of developed hydrodynamic cavitation in order to more intensively release silver ions, $\mathrm{U}[\mathrm{V}]-\mathrm{X}_{2}$.

The microbiological studies were conducted in an accredited laboratory of National Water Supply Company -Plovdiv. Test complex was accredited by EA "BSA" according to BDS EN ISO/IEC 17025:2006. In the carried out studies on the process of wastewater purifying from the APS with hydrodynamic cavitation, were obtained dependencies for the percentage of killed microorganisms, respectively - the degree of water disinfection.

Table 2. Natural values of factors

\begin{tabular}{|l|c|l|}
\hline \multirow{2}{*}{ Level } & $\mathrm{X}_{2}$ & $\mathrm{X}_{1}$ \\
\cline { 2 - 3 } & Voltage U [V] & $\begin{array}{l}\text { Time } \\
{[\mathrm{min}]}\end{array}$ \\
\hline Upper level (+) & 17 & 8 \\
\hline Lower level ( - ) & 27 & 2 \\
\hline Zero level & 22 & 5 \\
\hline Variation interval & 5 & 3 \\
\hline Code & $\mathrm{x}_{\mathbf{2}}=\frac{\mathrm{x}_{\mathbf{2}}-\mathbf{2 2}}{\mathbf{5}}$ & $\mathrm{x}_{\mathbf{1}}=\frac{\mathrm{x}_{\mathbf{1}} \mathbf{- 5}}{\mathbf{3}}$ \\
\hline
\end{tabular}

The silver ions separated in the cavitation zone also have bactericidal action. 


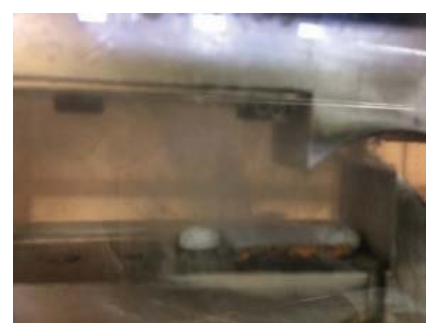

Fig. 5 Voltage apply process - Ag-plate $U=17 \mathrm{~V}$.

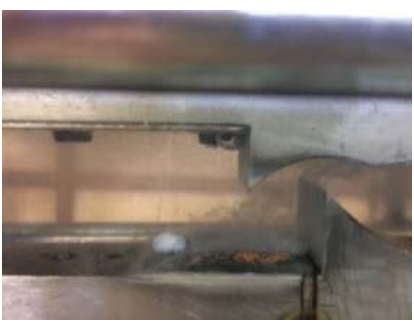

Fig. 6 Voltage apply process $-A g$-plate $U=27 \mathrm{~V}$

A higher percentage of microbiological purification of water was observed at the submitted voltage $\mathrm{U}=27 \mathrm{~V}$. Figures 5 and 6 illustrate the process of applying DC voltage to a silver plate. The plate was fixed exactly at the place of formation of the developed cavitation with the purpose of intensive separation of silver ions.

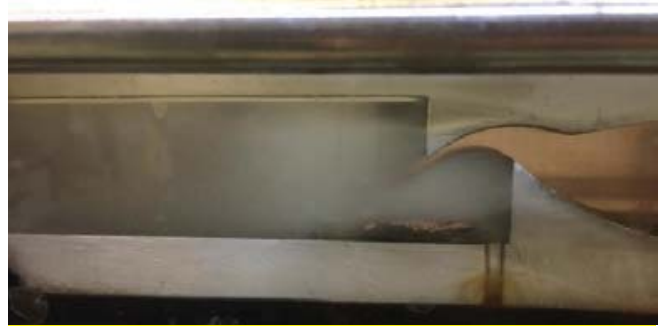

Fig. 7 Cavitator photo in a cavitation mode

Figure 7 presents a real photo of the cavitation phenomenon during the studies. Table 3 presents the values for the modification of the microbial number during the cavitation process for the different combinations of factor levels.

Table 3. Experiment Matrix

\begin{tabular}{|l|l|l|l|l|l|l|l|}
\hline № & $\mathrm{X} 0$ & $\mathrm{X} 1$ & $\mathrm{X} 2$ & $\mathrm{Y} 1$ & Y2 & Y3 & $\bar{Y}$ \\
\hline 1 & + & - & - & 5090 & 6128 & 5362 & 5526.7 \\
\hline 2 & + & - & + & 2612 & 3016 & 2918 & 2848.7 \\
\hline 3 & + & + & - & 1267 & 1427 & 1360 & 1351.3 \\
\hline 4 & + & + & + & 826 & 1000 & 1015 & 947 \\
\hline 5 & 0 & 0 & 0 & 1827 & 1100 & 1334 & 1420.3 \\
\hline
\end{tabular}

Table 4. Analysis of Variance for microbe number

\begin{tabular}{|l|l|l|l|l|l|}
\hline Source & Sum of Squares & $D f$ & Mean Square & F-Ratio & P-Value \\
\hline $\mathrm{X}_{1}$ :time & $2.76974 \mathrm{E} 7$ & 1 & $2.76974 \mathrm{E} 7$ & 64.59 & 0.0000 \\
\hline $\mathrm{X}_{2}:$ voltage & $7.12558 \mathrm{E} 6$ & 1 & $7.12558 \mathrm{E} 6$ & 16.62 & 0.0018 \\
\hline $\mathrm{X}_{1} \mathrm{X}_{2}$ & $3.87717 \mathrm{E} 6$ & 1 & $3.87717 \mathrm{E} 6$ & 9.04 & 0.0119 \\
\hline Total error & $4.71715 \mathrm{E} 6$ & 11 & 428832 & & \\
\hline Total (corr.) & $4.34174 \mathrm{E} 7$ & 14 & & & \\
\hline
\end{tabular}

The ANOVA table partitions the variability in microbe number into separate pieces for each of the effects. It then tests the statistical significance of each effect by comparing the mean square against an estimate of the experimental error. In this case, 3 effects have Pvalues less than 0.05 , indicating that they are significantly different from zero at the $95.0 \%$ confidence level (table 4). The R-Squared statistic indicates that the model as fitted 
explains $89.1353 \%$ of the variability in microbe number. The adjusted R-squared statistic, which is more suitable for comparing models with different numbers of independent variables, is $86.1722 \%$. The standard error of the estimate shows the standard deviation of the residuals to be 654.853. The mean absolute error (MAE) of 424.327 is the average value of the residuals. The Durbin-Watson (DW) statistic tests the residuals to determine if there is any significant correlation based on the order in which they occur in your data file. Since the P-value is greater than $5.0 \%$, there is no indication of serial autocorrelation in the residuals at the $5.0 \%$ significance level. After the analysis of the results, the following regression equation was obtained:

$$
\begin{aligned}
& \text { Microbial number }=2418.8-1519.3 * \mathrm{t}-770.6 * \mathrm{U}+568.4 * \mathrm{t} * \mathrm{U} \\
& \mathrm{Y}=2418.8-1519.3 \mathrm{X}_{1}-770.6 \mathrm{X}_{2}+568.4 \mathrm{X}_{1} \mathrm{X}_{2}
\end{aligned}
$$

Since all the independent variables are significantly different from zero, the regression equation seems to be adequate.

In equation (2), the numbers are the regression coefficients, and $X_{1}, X_{2}$ are the independent factors that influence the process. $\mathrm{Y}$ is a response function. The two input factors $\mathrm{X}_{1}$ (t-time), $\mathrm{X}_{2}$ (U-voltage) influence the process cavitational purification of water. The following graphs show the reflection areas and the isolines for the modification of the microbial number depending on $\mathrm{X}_{1}$ and $\mathrm{X}_{2}$. The nature of the resulting surface indicates the simultaneous strong impact of both factors: $\mathrm{X}_{1}$ - the cavitation time and $\mathrm{X}_{2}$ - U-voltage.

When the factors are at the upper level, they affect the process. The experiment was conducted under the same initial conditions, the samples of waste water were taken from the same place during two weeks at the outlet of a treatment plant after the sum of all secondary radial precipitators from the urban treatment plant. As the processing time $\left(\mathrm{X}_{1}\right)$ of the water purification increases, the microbial number decreases significantly.

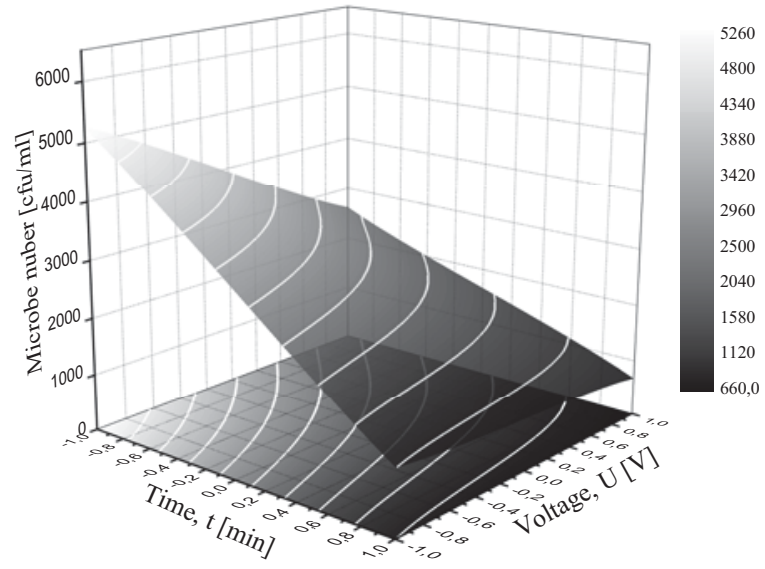

Fig. 8 Response surface and isolines for the modification of the microbial number depending on time and voltage.

Figure 8 presents the dependence of the number of killed microorganisms on the time and voltage factors that are set in the experiment. A strong reduction in the microbial number was observed at the beginning of the process up to the second minute of the experiment. Compared to the other factor $\left(\mathrm{X}_{1}-\right.$ time) the influence of $\mathrm{X}_{2}$ (voltage) is weaker, but it still is a significant factor that influences the research process. In order to achieve the optimal effect of the interaction and the influence of the process factors, $\mathrm{X}_{2}$ must be of a maximum value - at the upper level $(\mathrm{U}=27 \mathrm{~V})$. 
In order to be used in practice the obtained regression equation (3) has to be modificated in a natural form (4).

$$
\begin{aligned}
& \mathrm{Y}=2418.8-1519.3 * \mathrm{t}-770.6 * \mathrm{U}+568.4 * \mathrm{t} * \mathrm{U} \\
& \mathrm{Y}=2418.8-1519.3 * \frac{x_{1}-5}{3}-770.6 \frac{x_{2}-22}{\mathbf{5}}+568.4 * \frac{x_{1}-\mathbf{5}}{3} * \frac{x_{2}-22}{5}
\end{aligned}
$$

The positive influence of the combination of factors $\mathrm{X}_{1}$ and $\mathrm{X}_{2}\left(\mathrm{X}_{1}\right.$-time, $\mathrm{X}_{2}$-voltage $)$ was found during the analysis of the cavitation treatment process. In order to analyze the rate of purification of waste water, it is necessary to calculate the percentage of killed microorganisms for each carried out attempt $\left(\mathrm{Y}^{\prime}\right)$. Table 4 presents the values for the percentage of killed microorganisms during the process in each repetition of the experiments.

Tab. 4.Percentage of killed microorganisms

\begin{tabular}{|c|c|c|c|c|c|c|c|}
\hline № & $\mathrm{X} 0$ & $\mathrm{X} 1$ & $\mathrm{X} 2$ & $\mathrm{Y} 1$ & $\mathrm{Y} 2$ & $\mathrm{Y} 3$ & $\bar{Y}$ \\
\hline 1 & + & - & - & 32.54 & 18.7 & 28.9 & 26.7 \\
\hline 2 & + & - & + & 8783 & 85.9 & 86.3 & 86.7 \\
\hline 3 & + & + & - & 84.8 & 82.8 & 83.6 & 83.7 \\
\hline 4 & + & + & + & 91 & 89.1 & 88.9 & 89.7 \\
\hline 5 & 0 & 0 & 0 & 67.8 & 91.2 & 89.4 & 82.8 \\
\hline
\end{tabular}

With the obtained data, a plan matrix of the experiment was constructed and a $2^{2}$ full factor experiment was carried out. The influencing factors are the same as those in the analysis of the microbial number. The work was done following the algorithm described in section 4.2. The following regression equation was obtained:

Percentage of killed microorganisms $=73.97+15.07 * \mathrm{t}+16.54 * \mathrm{U}-13.44 * \mathrm{t} * \mathrm{U}$

$$
\mathrm{Y}^{\prime}=73.9+15.1 \mathrm{X}_{1}+16.5 \mathrm{X}_{2}-13.4 \mathrm{X}_{1} \mathrm{X}_{2}
$$

The modification in the percentage of killed microorganisms depending on the combination of the individual significant influence factors, obtained after processing the experimental data is presented in Figure 9.

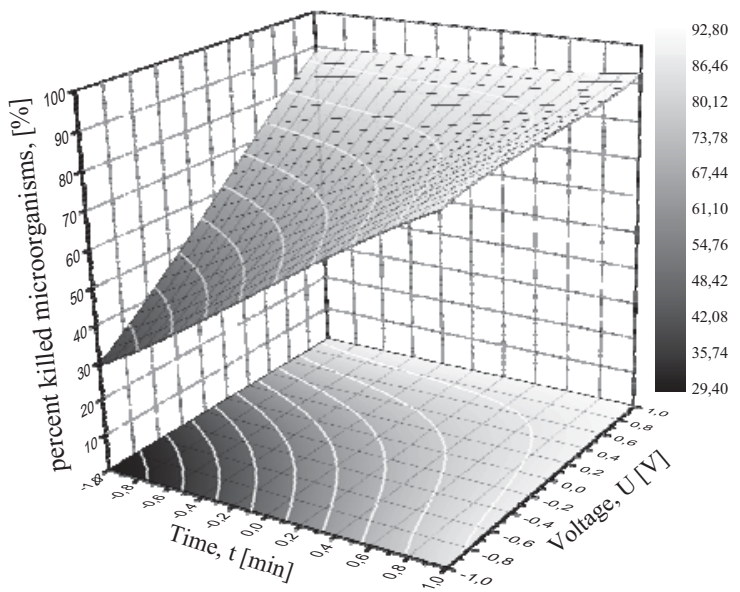

Fig. 9 Reflection surface and isolines about the modification in the percentage of killed microorganisms depending on time $\mathrm{X} 1$ and voltage $\mathrm{X} 2$

$\mathrm{Y}^{\prime}$ - in the graph expresses the percentage of killed microorganisms and the degree of wastewater purification. 


\section{Conclusions}

From the carried out experimental studies and the obtained regression equations (on the influence of factors on the percentage of killed microorganisms) the following conclusions can be drawn:

1. The most positive influence on the waste water purification process has the cavitation time $\mathrm{X}_{1}$. The average percentage of killed microorganisms in the second minute of cavitation processing is over $60 \%$. Then it increases gradually, reaching $80 \%$ in the fourth minute of processing. Further, the increase in the percentage of killed microorganisms is not as sharp as until the second minute of processing. After 6 minutes of treatment, $90-92 \%$ water purification is achieved, and in the end of the process ( 8 minute) it is $94-95 \%$. Maximum degree of purification in 8 minutes of water treatment is $94-95 \%$.

2. Combined positive influence has the combination of factors $X_{1} X_{2}-$ voltage and processing time.

3. The applied voltage in the cavitation zone $\left(\mathrm{X}_{2}\right)$ also has a positive impact. In this way the process of wastewater treatment is intensified and the percentage of killed microorganisms is increased.

4. The presented regression model describes the process of cavitation of wastewater treatment from microbiological contaminants and creates a methodology for its application. 5. The developed methodology presents a physical method for additional treatment of water discharged into reservoirs after treatment plants, which is reliable, economical and safe for both the user and the environment.

6. Cavitation treatment of wastewater can be intensified by placing a silver plate in the impact zone.

7. Cavitation treatment of wastewater can be intensified by the submission of DC voltage.

8. The obtained results show that it is possible to treat wastewater from an urban treatment plant with hydrodynamic cavitation.

\section{References}

1. Ordinance № 2 of March 22, 2005 On the design, construction and operation of water supply systems (2005)

2. Ordinance on Water No 9., State Gazette, 30 (2001)

3. D. Stoeva, Cavitation treatment of liquids, Dissertation, (2006)

4. A. Pandit, K.Jyoti, Hybrid cavitation methods for water disinfection, Biochemical Engineering Journal, 14 (2003)

5. P.Gogate, Cavitation: an auxiliary technique in wastewater treatment schemes, Adv. Environmental Research J., 6 (2002)

6. A. Pandit, Wastewater treatment: a novel energy efficient hydrodynamic cavitational thechnique, Ultrasonics Sonochemistry, 9 (2002)

7. A. Pandit, Water disinfection by acoustic and hydrodynamic cavitation, Biochemical engineering Journal, 7 (2001)

8. M. Angelov, Cavitation application for liquid treatment. Habilitation work "Professor", Plovdiv (2009)

9. A. Chakinala, P. R.Gogate, A. Burgess, D. Bremner, Intensification of hydroxyl radical production in sonochemical reactors, Ultrasonics Sonochemistry 14509 (2006)

10. P. Gogate, A. B. Pandit, Engineering design methods for cavitation reactors II: hydrodynamic cavitation, American Institute of Chemical Engineers Journal, 468 (2000) 
11. P.C. Sangave, A.B. Pandit, , Ultrasound pre-treatment for enhanced biodegradability of the distillery wastewater, Ultrasonics Sonochemistry 11, 197 (2004)

12. M. Sivakumar, Cavitation technology - A greener processing technique for the generation of pharmaceutical nanoemulsions, Ultrasonics Sonochemistry 21, 2069 (2014)

13. A. Terziev, A. Genbach, N. Jamankulova, I. Iliev, Study of fragile capillary-porous coatings in power installations, E3S Web Conf. Enviro 2018 - Sustainable Solutions for Energy and Environment, 85 (2019)

14. M. Angelov M, P.R. Raynov, J Model studies of the hydrodynamics of flow in corrugated tubes, Journal of Food and Packaging Science Technique and Technologies, 2, 274 (2013)

15. M. Angelov, The role of cavitation treatment to improve fluid quality, TU Sofia, Proceedings, Proceedings, Scientific Conference of the EMF, 2 (2012)

16. Y Tomita, P. B. Robinson, R. P. Tong, J. R. Blake, Growth and collapse of cavitation bubbles near a curved rigid boundary, Fluid Mechanics, 466259 (2002)

17. N. Krystev, P. Kostov, D. Angelova, Numerical Simulation of Gas Fuel Burning in Injected Swirled Jet, National Committee for the Theory of Machines and Mechanisms, Thermal Eng. J., 8, 7 (2014) 